University of Nebraska - Lincoln

DigitalCommons@University of Nebraska - Lincoln

April 1993

\title{
Multiplet fine structure in the photoemission of the gadolinium and terbium $5 p$ levels
}

\author{
B.T. Thole \\ University of Groningen \\ X.D. Wang \\ lowa State University, Ames, lowa \\ B.N. Harmon \\ lowa State University, Ames, lowa \\ Dongqi Li \\ University of Nebraska-Lincoln \\ Peter A. Dowben \\ University of Nebraska-Lincoln, pdowben@unl.edu
}

Follow this and additional works at: https://digitalcommons.unl.edu/physicsdowben

Part of the Physics Commons

Thole, B.T.; Wang, X.D.; Harmon, B.N.; Li, Dongqi; and Dowben, Peter A., "Multiplet fine structure in the photoemission of the gadolinium and terbium 5p levels" (1993). Peter Dowben Publications. 58.

https://digitalcommons.unl.edu/physicsdowben/58

This Article is brought to you for free and open access by the Research Papers in Physics and Astronomy at DigitalCommons@University of Nebraska - Lincoln. It has been accepted for inclusion in Peter Dowben Publications by an authorized administrator of DigitalCommons@University of Nebraska - Lincoln. 


\title{
Multiplet fine structure in the photoemission of the gadolinium and terbium $5 p$ levels
}

\author{
B. T. Thole \\ Department of Chemical Physics, University of Groningen, Nijenborgh 16, 9747, A.G. Groningen, The Netherlands \\ X. D. Wang and B. N. Harmon \\ Ames Laboratory and the Department of Physics and Astronomy, Iowa State University, Ames, Iowa 50011
}

Dongqi Li and P. A. Dowben

Department of Physics, Syracuse University, Syracuse, New York 13244-1130

(Received 17 September 1992)

\begin{abstract}
Fine structure is observed in the photoemission of the gadolinium and terbium $5 p$ levels. The $5 p$ levels are split into multiplets due to spin-orbit splitting and to Coulomb and exchange interactions with the $f$ shell. The calculated theoretical spectra are in good agreement with the experimental results.
\end{abstract}

Rare-earth metals are well known for exhibiting pronounced multiplet splittings of the $4 d$ levels, ${ }^{1-4} 4 f$ levels, ${ }^{4-8}$ and the $4 s$ and $5 s$ levels. ${ }^{4,9,10}$ Only recently has multiplet splitting in the photoemission spectra of the gadolinium and terbium $5 p$ levels been reported. ${ }^{11-13}$ For emission just above threshold, the multiplet level intensities have been demonstrated to be sensitive to the structural environment of the atom. ${ }^{12}$ Indeed, the multiplet intensities are sensitive to magnetic ordering in angle-resolved photoemission giving rise to an effect similar to, but much larger than, magnetic linear dichroism far above threshold. ${ }^{14}$ Here we consider experiments on thick $\mathrm{Gd}$ and $\mathrm{Tb}$ films where the multiplet structure is much less sensitive to the light polarization and magnetic ordering. Understanding this "stable" multiplet structure is an important first step for investigating what might cause the light polarization sensitivity observed for very thin films.

The angle-resolved photoemission experiments were carried out in a UHV system described elsewhere ${ }^{15}$ on a 6 $m$ toroidal grating monochromator at the Synchrotron Radiation Center in Stoughton, Wisconsin. The photoelectrons were collected normal to the surface. The light incidence angle was $70^{\circ}$ off normal. The proton energy was 50 or $60 \mathrm{eV}$ and the combined energy resolution for the photoemission was $0.150 \mathrm{eV}$.

The gadolinium films were deposited at room temperature onto $\mathbf{W}(110)$ substrates, using established techniques that provide clean rare-earth films. ${ }^{14-16}$ The gadolinium films on W(110) show sharp low-energy electron diffraction patterns indicating the well-ordered growth with the $(0001)$ orientation of the hexagonal gadolinium film long the surface normal.

The photoemission spectrum of the gadolinium $5 p$ features for a thick (30 $\AA$ ) film on W(110) is shown in Fig. 1. Figure $1(a)$ is one of the typical spectra which includes two main features with binding energies of about 21 and $28 \mathrm{eV}$. The $21 \mathrm{eV}$ feature exhibits fine structure which can be well fitted with four Gaussian peaks with binding energies of $20.3 \pm 0.1 \mathrm{eV}, 20.9 \pm 0.1 \mathrm{eV}, 22.0 \pm 0.2 \mathrm{eV}$, and $23.4 \pm 0.3 \mathrm{eV}$. The $5 p$ levels for terbium are very similar
[Fig. 1(b)]. The main features for terbium have binding energies of 22 and $28 \mathrm{eV}$, respectively. Though not as clear as for gadolinium, the $22 \mathrm{eV}$ feature also contains structure with peaks at binding energies of 20.8 and 22.1 $\mathrm{eV}$ as discussed in detail elsewhere. ${ }^{1}$

As expected, the $5 p$ photoemission fine structure due to multiplet splittings is a result of the unfilled $4 f$ levels. Multiplet structures can be understood as a final-state effect in photoemission. After one $5 p$ electron is excited, the hole left behind will have an electrostatic interaction (Coulomb and exchange) with the electrons in unfilled subshells to form different final states. This effect has been well studied on core levels of transition metals and rare earths. ${ }^{8-10,17-19}$ Most of the multiplet studies have only considered the exchange interaction between the unpaired spins which results in two main features characterized by spin parallel or antiparallel to that of the unpaired electrons. For $s$ levels, there is no spin-orbit interaction since they have no orbital momentum $(l=0)$. For $p$ levels $(l=1)$, spin-orbit interaction can be ignored only when it is much weaker than the exchange interaction between the hole and the other electrons, that is, in the $L-S$ coupling limit.

A large energy separation $(\sim 7 \mathrm{eV})$ of the two main

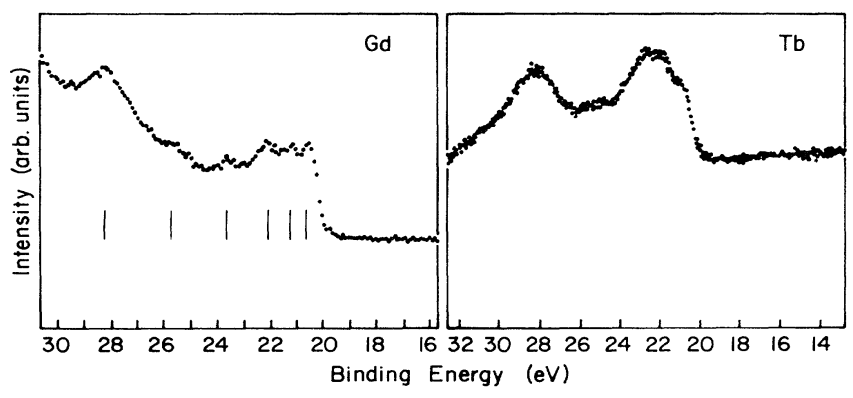

FIG. 1. (a) A typical Gd $5 p$ level photoemission spectrum with photon energy $50 \mathrm{eV}$. (b) $\mathrm{Tb} 5 p$ level photoemission spectrum with photon energy of $60 \mathrm{eV}$ (taken from Ref. 11).

(c) 1993 The American Physical Society 
photoemission features has been observed for the rareearth $5 p$ levels. . $^{41,14,15,20}$ This is mainly a result of spinorbit coupling but spin-orbit interactions alone cannot be considered in understanding the spectra of the $5 p$ levels, since they would produce only two features $\left(5 p_{1 / 2}\right.$ and $\left.5 p_{3 / 2}\right)$.

For gadolinium the main features of the spectrum can be understood when we consider the $f^{7}$ electrons as being frozen in the ${ }^{8} S_{7 / 2}$ state. With the $L-S$ coupling scheme, the possible multiplets for gadolinium are easily determined. The ground-state configuration of the gadolinium atom is $4 f^{7} 5 s^{2} 5 p^{6} 5 d^{1}$. Since the interaction of the $5 d$ with the $5 p$ and $4 f$ electrons is small we will only consider the $5 p$ hole and the $4 f^{7}\left({ }^{8} S_{7 / 2}\right)$ electrons. Photoemission of one of the $5 p$ electrons leaves an ion in the $5 p^{5} 4 f^{7}$ configuration. Since $l=1, s=1 / 2$ for ${ }^{2} P$ and $L^{\prime}=0$ and $S^{\prime}=7 / 2$ for ${ }^{8} S, L-S$ coupling of $5 p^{5}\left({ }^{2} P\right)$ and $4 f^{7}\left({ }^{8} S\right)$ results in ${ }^{7} P_{4,3,2}$ and ${ }^{9} P_{3,4,5}$ final states. Without considering spin-orbit coupling, the two main features should be assigned as ${ }^{7} P$ and ${ }^{9} P$ with the latter one at the lower binding energy. If weak spin-orbit coupling exists, the degeneracy of the different $J$ values should be lifted and the ${ }^{9} P$ and ${ }^{7} P$ should each split into three features as schematically indicated in Fig. 2.

Alternatively, the multiplet splitting can also be described in $j-J$ coupling. Since the spin-orbit coupling is strong for the heavy rare-earth metals terbium and gadolinium, as noted above, we can assign the two envelopes of intensity (containing several finer features) as the $5 p_{1 / 2}$ and $5 p_{3 / 2}$ based levels with the latter one at the lower binding energy (Fig. 1). The relatively weaker exchange interaction of the $5 p$ hole with $4 f$ electrons will split

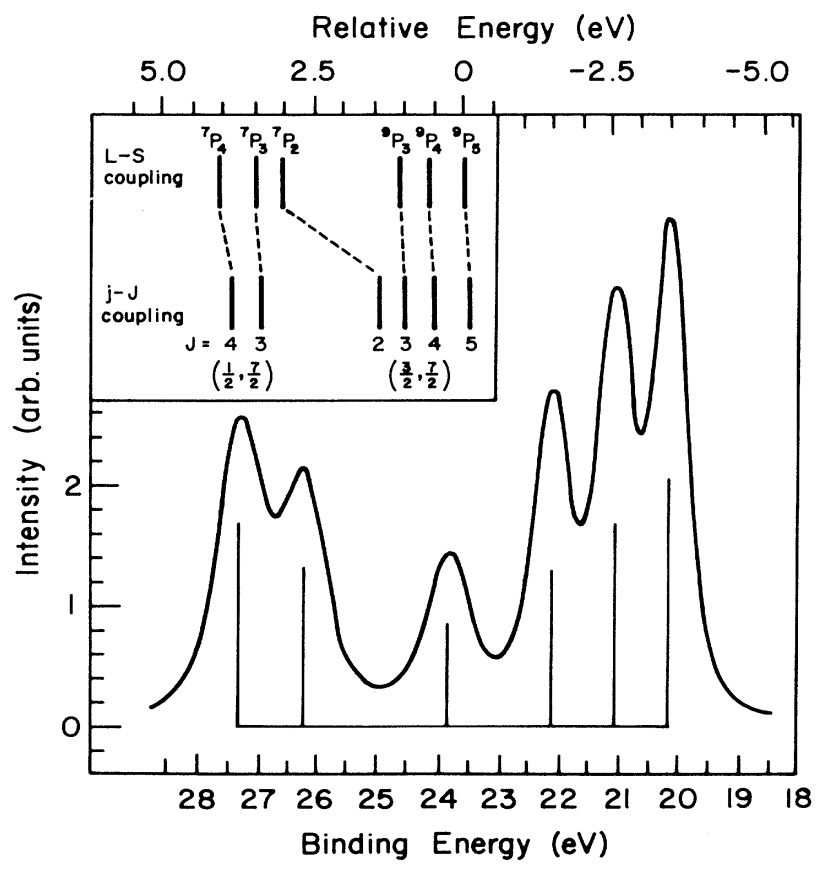

FIG. 2. Results for $4 f-5 p$ interaction upon the $5 p$ photoemission for gadolinium, calculated for the $4 f^{7}$ remaining frozen in the ${ }^{8} S_{7 / 2}$ configuration in the final state. The inset shows the order of different $J$ terms in $L-S$ and $j-J$ coupling schemes. these two states. The $j=3 / 2$ term couples with the ${ }^{8} S_{7 / 2}$ to form four levels with $J$ valuess $(3 / 2,7 / 2)_{5},(3 / 2$, $7 / 2)_{4},(3 / 2,7 / 2)_{3}$; and $(3 / 2,7 / 2)_{2}$. This is consistent with the fourfold splitting observed in experiment for the $5 p_{3 / 2}$ envelope.

To determine quantitatively the multiplet fine structure, we evaluated the atomic spectra for all $5 p^{6} 4 f^{7}$ to $5 p^{5} 4 f^{7}$ transitions. The calculations were performed using Cowan's multiplet program with parameters calculated by his atomic Hartree-Fock program with relativistic corrections. This program has been successfully applied to study the multiplet structure in the $5 p$ and $5 s$ x-ray photoemission of several actinides. ${ }^{21}$ The electrostatic and exchange parameters for the $\mathrm{Gd}^{3+}$ ground state are the same as those given by Thole et al. ${ }^{23}$ The parameters for the $5 p^{5} 4 f^{7}$ state are $F^{2}(f f)=11.83 \mathrm{eV}, F^{4}(f f)=7.44$ $\mathrm{eV}, F^{6}(f f)=5.35 \mathrm{eV}, F^{2}(f p)=5.43 \mathrm{eV}, G^{2}(f p)=2.73$ $\mathrm{eV}, G^{4}(f p)=2.13 \mathrm{eV}, \zeta_{f}=0.201 \mathrm{eV}$, and $\zeta_{p}=2.974 \mathrm{eV}$. The values of the $F$ and $G$ parameters are $80 \%$ of the Hartree-Fock values. This $20 \%$ reduction has been found in numerous calculations to provide a simple means to simulate the effects caused by intra-atomic correlation. $^{21,22}$ The spin-orbit parameters have the full Hartree-Fock values.

We first performed calculations freezing the configuration of the $4 f$ shell to ${ }^{8} S$ and this yielded a reasonable spectrum as shown in Fig. 2. This spectrum is close to the $j-J$ coupling scheme sketched in Fig. 2 . The relative energies for the multiplets are not in complete agreement with experiment, however. Since the $p-f$ interactions are rather strong, the $4 f$ shell does not remain in the ${ }^{8} S$ state, but other $L S$ terms are mixed in. There-

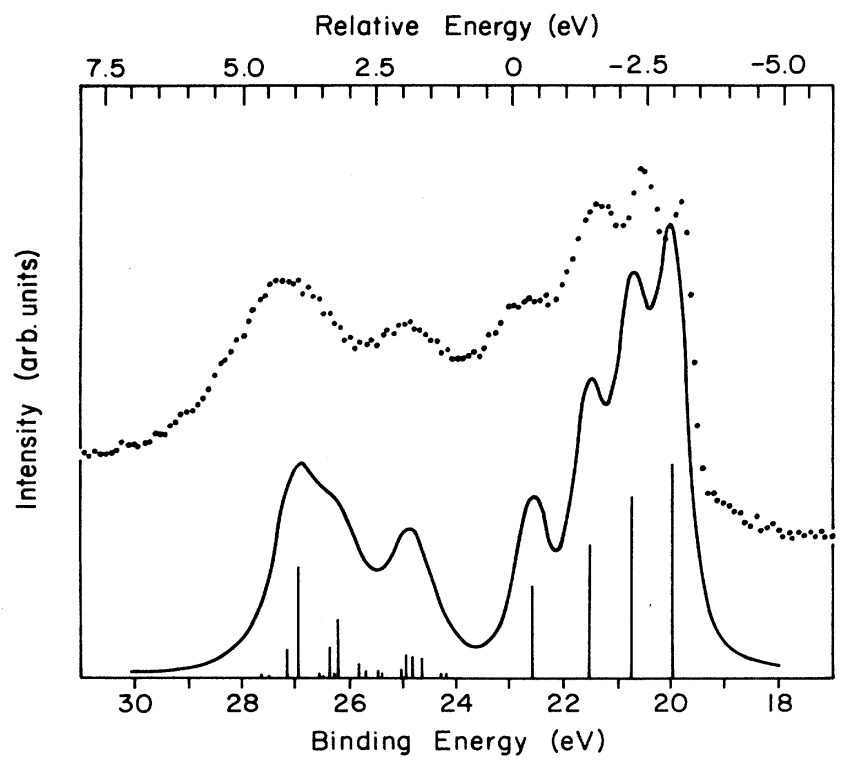

FIG. 3. Calculated results for the $4 f$ induced $5 p$ multiplet splittings shown as a solid line for gadolinium. The multiplet levels are also shown. The experimental data for the $5 p$ corelevel multiplets of gadolinium (0001) are also shown for a photon energy of $60 \mathrm{eV}$ and all photoelectrons collected normal to the surface. The Gd (0001) crystal was prepared by depositing $30 \AA$ of gadolinium on W(110). 


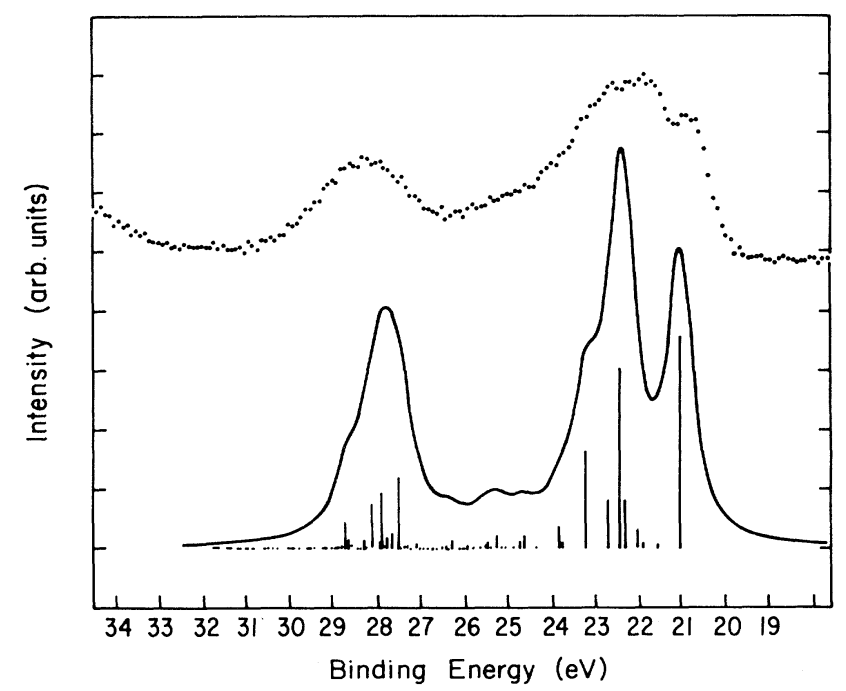

FIG. 4. Calculated result for all the $f^{8}\left({ }^{7} F_{6}\right) \rightarrow 5 p^{5} f^{8}$ transitions for $\mathrm{Tb}^{3+}$ is shown as a solid line. The experimental data for the $5 p$ core-level multiplets of $\mathrm{Tb}$ are shown for a photon energy of $60 \mathrm{eV}$ and all photoelectrons were collected normal to the surface. The Tb film was prepared by depositing $80 \AA$ of $\mathrm{Tb}$ on $\mathrm{Ni}(111)$ at $150 \mathrm{~K}$.

fore, in a second calculation we included all possible multiplet states of the $4 f^{7}$ shell with the $5 p$ hole. The result is shown in Fig. 3, along with the experimental data. The theoretical multiplet lines have been broadened by a Lorentz line shape (corresponding to the $5 p$ hole lifetime broadening) of about $0.2 \mathrm{eV}$ and then the resulting spectrum was fully convoluted with a Gaussian line shape with a standard deviation of $0.22 \mathrm{eV}$.

This calculated result is in good agreement with the data for gadolinium. States other than ${ }^{8} S$ clearly need to be included to obtain this good agreement with the observed multiplet structure. The fact that an isolated ionic model was able to give such good results for the multiplet splittings far above threshold would suggest that any additional splittings arising from crystal-field effects and/or magnetic ordering are small for these thick films.

Terbium has a ground-state configuration of $4 f^{8} 5 s^{2} 5 p^{6} 5 d^{1}$, which is similar to that of gadolinium, although terbium has only six unpaired $4 f$ holes instead of the seven for gadolinium. For terbium, correct energy positions of the peaks can again be obtained by full multiplet calculation as seen in Fig. 4. The calculated intensities show a large discrepancy with experiment, however. It would seem that the linewidth of all peaks, except the lowest energy one, is much larger than the value we used. Indeed, preliminary calculations of the lifetimes of the peaks due to the Auger decay $5 p^{5} f^{n} \rightarrow f^{n-2} e$ where $e$ is a continuum electron, shows the tendency of producing a much (2-3 times) longer lifetime for the lowest energy peak than for the other peaks. This effect is also observable in the Gd spectrum.

In conclusion, we can say that the multiplet structure in the $5 p$ photoemission of $\mathrm{Gd}$ and $\mathrm{Tb}$ far above threshold is produced by Coulomb, exchange, and spin-orbit splitting of the $5 p^{5} 4 f^{n}$ final states. The linewidths of the peaks are different due to different Auger decay probabilities.

X. D. Wang is grateful to P. Carra and the staff at ESRF for their hospitality during the course of this work. Ames Laboratory is operated for the U.S. Department of Energy by Iowa State University under contract number W-7405-Eng-82. This work was supported by the U.S. D.O.E. We would also like to thank Jiandi Zhang and M. Onellion for their technical assistance.
${ }^{1}$ C. S. Fadley and D. A. Shirley, Phys. Rev. A 2, 1109 (1970).

${ }^{2}$ S. P. Kowalczyk, N. Edelstein, F. R. McFeely, L. Ley, and D. A. Shirley, Chem. Phys. Lett. 29, 491 (1974).

${ }^{3}$ J. L. Dehmer, A. F. Starace, U. Fano, J. Sugar, and J. W. Copper, Phys. Rev. Lett. 26, 521 (1971).

${ }^{4}$ B. P. Padalia, W. C. Lang, P. R. Norris, L. M. Watson, and D. J. Fabian, Proc. R. Soc. London, Ser. A 354, 269 (1977).

${ }^{5}$ R. Kammerer, J. Barth, F. Gerken, A. Flodström, and L. I. Johansson, Solid State Commun. 41, 435 (1982).

${ }^{6}$ Y. Baer and G. Busch, J. Electron Spectrosc. Relat. Phenom. 5, 611 (1974).

${ }^{7}$ J. K. Lang, Y. Baer, and P. A. Cox, J. Phys. F 11, 121 (1981).

${ }^{8}$ P. A. Cox, J. K. Lang, and Y. Baer, J. Phys. F 11, 113 (1981).

${ }^{9}$ R. L. Cohen, G. K. Wertheim, A. Rosencwaig, and H. J. Guggenheim, Phys. Rev. B 5, 1037 (1972).

${ }^{10}$ F. R. McFeely, S. P. Kowalczyk L. Ley, and D. A. Shirley, Phys. Lett. 49A, 301 (1974).

${ }^{11}$ Dongqi Li, P. A. Dowben, and M. Onellion, in Magnetic Thin Films, Surfaces and Multilayers, edited by S. S. P. Parkin, H. Hopster, J.-P. Renard, T. Shinjo, and W. Zinn, MRS Symposia Proceedings No. 231 (Materials Research Society, Pittsburgh, 1992), p. 107.

${ }^{12}$ Dongqi Li, C. Hutchings, P. A. Dowben, Rong-Tzong Wu, C.
Eiwang, M. Onellion, A. B. Andrews, and J. L. Erskine, J. Appl. Phys. 70, 6062 (1991).

${ }^{13}$ R. I. R. Blyth, A. J. Patchett, S. S. Dhesi, R. Cosso, and S. D. Barrett, J. Phys. Condens. Matter 3, s287 (1991); P. A. Dowben, Dongqi Li, and M. Onellion, ibid. 4, 7021 (1992); J. A. Scarfe, A. R. Law, H. P. Hughes, J. A. C. Bland, G. M. Roe, and A. D. Walker, Phys. Status Solidi B 171, 377 (1992).

${ }^{14}$ P. A. Dowben, D. LaGraffe, Dongqi Li, A. Miller, Ling Zhang, L. Dottl, and M. Onellion, Phys. Rev. B 43, 3171 (1991).

${ }^{15}$ P. A. Dowben, D. LaGraffe, and M. Onellion, J. Phys. Condens. Matter 1, 6751 (1989).

${ }^{16}$ Dongqi Li, Jiandi Zhang, P. A. Dowben, and M. Onellion, Phys. Rev. B 45, 7272 (1992).

${ }^{17}$ C. S. Fadley, D. A. Shirley, A. J. Freeman, P. S. Bagus, and J. V. Mallow, Phys. Rev. Lett. 23, 1397 (1969).

${ }^{18}$ B. Hermsmeier, C. S. Fadley, M. O. Krause, J. Jimenez-Mier, P. Gerard, and S. T. Manson, Phys. Rev. Lett. 61, 2592 (1988).

${ }^{19}$ S. P. Kowalczyk, L. Ley, F. R. McFeely, and D. A. Shirley, Phys. Rev. B 11, 1721 (1975).

${ }^{20}$ G. K. Wertheim, R. L. Cohen, A. Rosencwaig, and H. J. Guggenheim, in Electron Spectroscopy, edited by D. A. Shirley 
(North-Holland, Amsterdam, 1972), p. 813.

${ }^{21}$ R. Cowan, J. Opt. Soc. Am. 58, 808 (1968); Theory of Atomic Structure and Spectra (University of California, Berkeley, 1981); M. Boring, R. D. Cowan, and R. L. Martin, Phys. Rev.
B 23, 445 (1981).

${ }^{22}$ B. T. Thole, G. van der Laan, J. C. Fuggle, G. A. Sawatsky, R. C. Karnatak, and J. M. Esteva, Phys. Rev. B 32, 5107 (1985). 